\title{
FRACTAL MHD WAVES IN THE SOLAR ATMOSPHERE
}

\section{LUIGI NOCERA}

Istituto di Fisica Atomica e Molecolare - CNR, Via Giardino 7, I-56127 Pisa

ABSTRACT Nonlinear MHD waves forced into a bounded cavity exhibit structural instability of their equilibria and periodic orbits as the forcing varies. For some values of the forcing the computed Poincare maps, Lyapunov exponents and phase-space correlation reveal that the waves become chaotic; the Haussdorff dimension of the waves' attracting set lies between its correlation dimension $(\approx 2.2)$ and its information dimension $(\approx 2.25)$.

\section{INTRODUCTION}

MHD waves in the solar atmosphere are prone to structural instability, defined as their ability to move from one dynamical equilibrium (a stationary oscillatory state) to another, as the former experiences an arbitrarily small perturbation.

In special occasions the partial differential equations for wave propagation can be reduced to ordinary differential equations (ODEs) for a few modes (see e.g. the lumped parameter model of Stepanov and Tsap in these proceedings). Then Classical Mechanics - jointly with numerical techniques - offers an excellent framework for the analysis of structural instability and it has been profitably used in several circumstances: see e.g. the work by Hada et al. (1990) who tested the structural stability of two-fluid, forced, dissipative Alfvén waves modelled by a Vector Derivative Nonlinear Schrödinger equation reduced to two ODEs.

Nocera and Priest (1991) found MHD waves in viscous, magnetized, forced cavities (Fig. 1) to be bistable as the forcing frequency varies. Here we report that they are prone to other structural instabilities (subharmonic bifurcations, e.g. Guckenheimer and Holmes 1983) and exhibit chaotic behaviour.

\section{BASIC EQUATIONS}

Let $\sqrt{\varepsilon} Z_{1}$ « 1 and $\omega$ be the velocity (normalized to the Alfvén speed) and the frequency of the Alfven wave at the boundaries of the cavity of Fig. 1; let p, q, r, s, $\sigma, \Phi$ be complex constants depending on the plasma $\beta$ and compressive Reynolds number $\mathrm{Re}_{0}$; let $B_{0}$,

$$
\delta B_{z}(x, t)=\sqrt{ } \varepsilon B_{0} \sum_{\mathrm{m}=1}^{\infty} H_{\mathrm{m}}(t) \exp (-i \mathrm{~m} \omega t) \sin (\mathrm{m} x)+\text { c.c., } H_{1}(0)=A+Z_{1}
$$

be the equilibrium, the perturbation magnetic fields in the cavity and the initial condition for $H_{1}$, c.c. denoting the complex conjugate and $A$ an arbitrary complex constant. Then, if $\delta \omega=\left(\omega^{2}-1\right) / 2 \omega$ is the frequency detuning, we find

$$
d H_{1} / d t=i \delta \omega\left(H_{1}-Z_{1}\right)+\varepsilon\left(\mathrm{p}\left|H_{1}\right|^{2}+\left.\mathrm{q}^{\mid H_{3}}\right|^{2}\right) H_{1}+\varepsilon \mathrm{r} \overline{H_{1}}{ }^{2} H_{3},
$$


Thus $D_{\mathrm{H}}$ is itself fractional and the limit set of the flow in Fig. 5a is fractal.
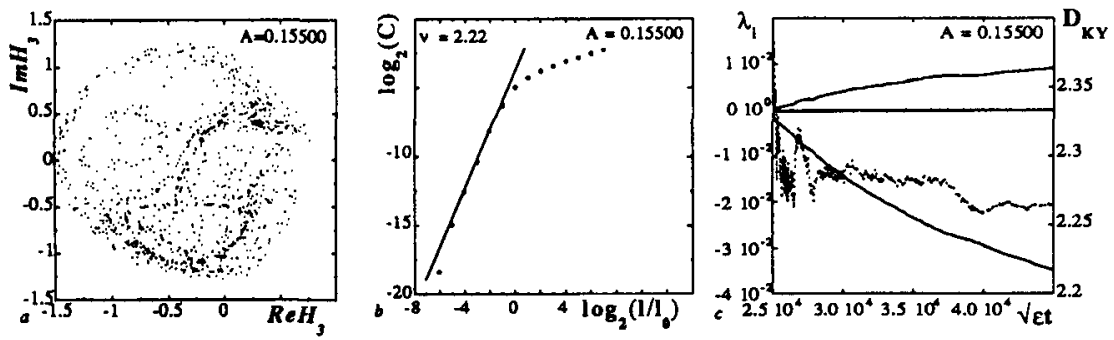

Fig. 5. The Poincare map (a), the phase-space correlation $C(b)$ and the four Lyapunov exponents $\lambda_{i}(\ldots)$ and information dimension $D_{K Y}(\ldots)$ (c) for the chaotic solution of (2). Parameters as in Fig. 3, but $A=0.155$.

\section{CONCLUSIONS}

The study of coronal loop dynamics and its relevance to flares has now entered a non linear phase. In this context, we have investigated the properties of waves in such structures, approximated as homogeneous cavities forced at the boundaries by photospheric motions. Varying the forcing's frequency, bistability of the waves' amplitude occurs and the energy released during a bistable transition is of practical use for coronal heating. Varying the forcing's amplitude, we observe subharmonic bifurcations and chaotic behaviour of the wave's amplitude: this provides an insight in the onset of turbulence, which is instrumental to both coronal heating and particle acceleration.

The normal form of the cavity's Duffing oscillations coincides with the one found by Hada et al. (1990) and this encourages the conjecture that general underlying properties may exist for waves in plasmas.

\section{ACKNOWLEDGEMENTS}

We are grateful to the Italian National Research Council for financial support.

\section{REFERENCES}

Grassberger, P., Procaccia, I. 1983 Physica 9D, 189.

Guckenheimer, J., Holmes, P. 1983 Nonlinear Oscillations, Dynamical Systems, and Bifurcations of Vector Fields. Springer Verlag, Berlin.

Hada, T., Kennel, C. F., Buti, B., Miølhus, E. 1990, Phys. Fluids B2, 2581.

Kaplan, J. L., Yorke, J. A. 1979 Functional Differential Equations and the Approximation to Fixed Points: Lecture Notes in Mathematics, Vol. 730, Peitgen H. O., Walther H. O. eds. Springer, Berlin, p. 204.

Nocera, L., Priest, E. R. 1991 J. Plasma Physics 46, 153. 


$$
\alpha=u^{3}\left\{u \pm\left[1-\xi^{2}\left(u^{2}-1\right)\right]^{1 / 2}\right\} /\left(u^{2}-1\right),
$$

where $\mathrm{u}=\left|H_{1}\right|$. These are represented in Fig. 4 for three values of viscosity.
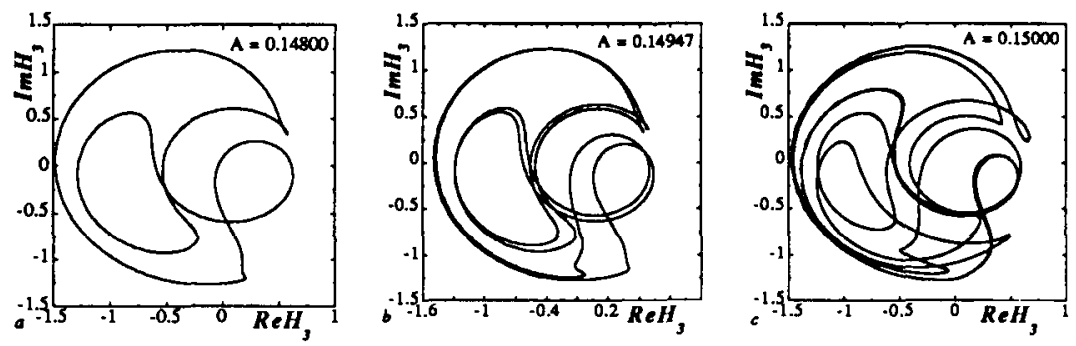

Fig. 3. Period 3, 6 and 12 orbits of (2). Parameters as in Fig. 2, but Re $e_{0}=$ 1000 and $A \neq 0$.
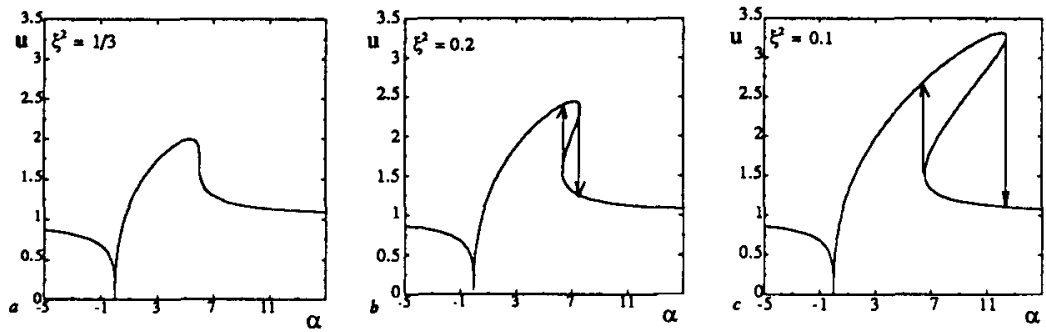

Fig. 4. Equilibria of (2a) according to (5). Arrows denote bistable transitions.

When $\mathrm{Re}_{0}$ is large, the maximum average volumetric power released during the downward transition of Fig. 4 is (Nocera and Priest 1991)

$$
W_{\max }=\frac{1}{3} \frac{\pi}{64} \varepsilon^{2} \operatorname{Re}_{0}^{3} B_{0}^{2} /\left(8 \pi \tau_{A}\right) \quad \text { at } \beta=1 \pm 1 / \sqrt{ } 3 .
$$

Here $\tau_{\mathrm{A}}$ is the Alfvén transit time over the cavity if Fig. 1 and $\mathrm{B}_{0}^{2} /\left(8 \pi \tau_{\mathrm{A}}\right)$ is the order of magnitude of the volumetric power released in an ideal MHD instability: due to the factor $\operatorname{Re}_{0}^{3},(6)$ compares well with that, despite the nonlinear factor.

\section{ONSET OF CHAOS}

When $A$ in eq. (1) approaches 0.155 , the wave flow enters a chaotic state (Fig. 5). A feature of the chaotic flow is one positive Lyapunov exponent and a fractional value of the Haussdorff dimension $D_{\mathrm{H}}$ of its limit set. A lower bound (the correlation dimension $v$ of Grassberger and Procaccia 1983) and an upper bound (the information dimension $D_{\mathrm{KY}}$ of Kaplan and Yorke 1979) to $D_{\mathrm{H}}$ are:

$$
v=2.22<D_{\mathrm{H}}<2.25=D_{\mathrm{KY}} \text {. }
$$




$$
d H_{3} / d t=3 i \delta \omega H_{3}+\varepsilon\left(\mathbf{s}\left|H_{1}\right|^{2}+\sigma\left|H_{3}\right|^{2}\right) H_{3}+\varepsilon \Phi H_{1}^{3} .
$$

Fig. 1. MHD waves propagate in a homogeneous tube of magnetized plasma whose boundaries move parallel to each other allowing for neither compressive nor slip motions.

\section{DUFFING OSCILLATIONS}

When the frequency detuning is $O(\sqrt{ } \varepsilon)$ (loose resonance) define

$$
\sqrt{ } \varepsilon \Delta \omega=\delta \omega .
$$

The system (2) becomes autonomous when $A=0$ and conservative when $\mathrm{Re}_{0} \rightarrow \infty$. In this case it can be derived from the Hamiltonian (the reduced Duffing Hamiltonian) whose energy levels are shown in Fig. 2: three continuous families of elliptic orbits exist each with different energy $E_{\alpha}$ and a period $T_{\alpha}$ which tends to $\infty$ as the orbits approach the double homoclinic loop (energy $E_{0}$ ). The study of the numerically computed Melnikov's function reveals that only those orbits whose period is an integer multiple of $2 \pi / \Delta \omega$ survive the introduction of forcing $(A \neq 0)$ and dissipation $\left(\operatorname{Re}_{0}<\infty\right)$. This is consistent with the results for the Duffing oscillator (e.g. Guckenheimer and Holmes 1983). Fig. 3 shows the period 3, 6 and 12 orbits.

\section{VAN DER POL OSCILLATIONS}

When the frequency detuning is $O(\varepsilon)$ (sharp resonance) define

$$
\varepsilon \alpha=\delta \omega, \xi=|R e p| /|l m p|
$$
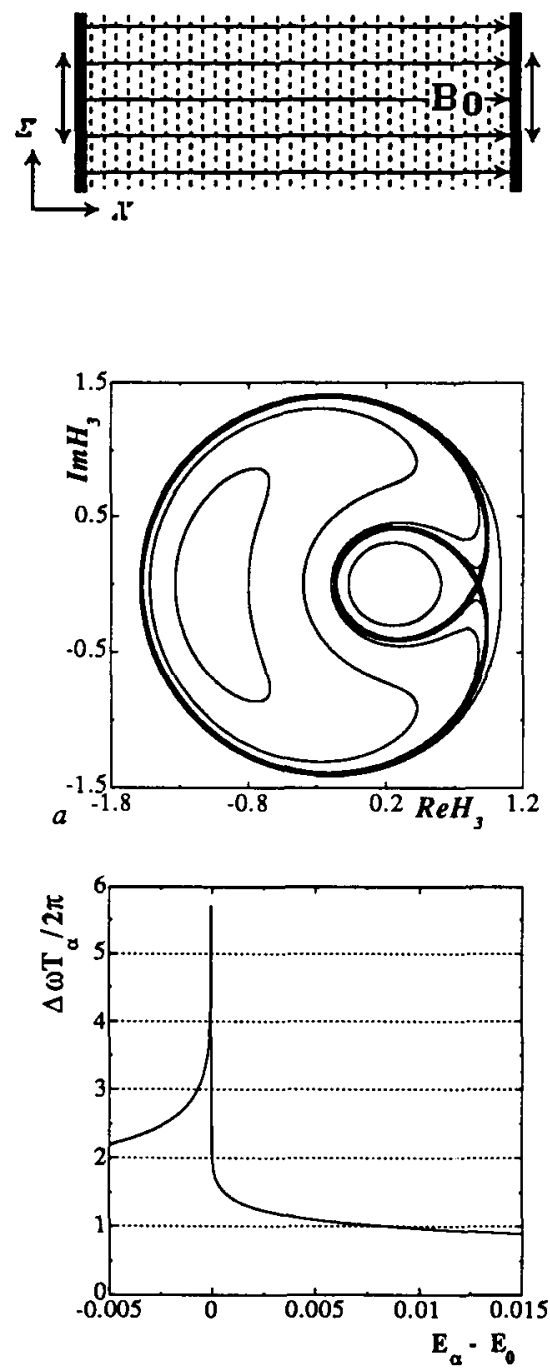

Fig 2. The energy levels, the double homoclinic loop (boldface) and the elliptic orbits ( $a$ ) and their periods ( $b$ ) for the conservative $\left(A=0, \operatorname{Re}_{0} \rightarrow\right.$ $\infty)$ system (2). $\beta=0.7, \sqrt{\varepsilon}=0.15$, $\Delta \omega=0.26, Z_{1}=0.6$.

Then to leading order, equation (2a) admits the stationary solutions 